\title{
Resolution Scalable Image Coding with Dyadic Complementary Rational Wavelet Transforms
}

\author{
F. Petngang, R.M. Dansereau, and C. Joslin \\ Department of Systems and Computer Engineering, \\ Carleton University, Ottawa, Ontario, Canada \\ rdanse@sce.carleton.ca, chris_joslin@carleton.ca
}

\begin{abstract}
Traditional wavelet-based image coders use dyadic multiresolution decompositions, resulting in spatial resolutions that are also dyadic in scale, i.e., powers of $1 / 2$ the original resolution. In this paper, we increase the set of decodable spatial resolutions beyond dyadic scales by introducing a two-dimensional wavelet decomposition made of combining wavelet transforms of non-integer dilation factors. We describe how the proposed wavelet decomposition can produce spatial resolutions related to the original resolution by dyadic and non-dyadic ratios, and present how the resulting subband structure can be made compatible with zerotree-based subband coders.
\end{abstract}

Keywords: Image compression, dyadic and non-dyadic spatial scalability, rational dilation wavelet transform.

\section{Introduction}

In image or video coding, the notion of scalability designates the idea that an image/video can be compressed once and decompressed in various ways to produce images of different visual quality, different spatial resolutions, or videos of different temporal resolutions. With the increasing diversity of media, different display terminal size, frame rates, and bandwidth, development of codecs with support for scalability on multiple dimensions provides an efficient way to accommodate various users from one single compressed file.

From the perspective of spatial scalability, multi-resolution decompositions using wavelet transforms are a natural choice. The successive sizes of the approximation subband produced during the wavelet decomposition constitute the native spatial resolutions available at the decoding side of a wavelet-based image/video compression system. As traditionally defined, a two-dimensional wavelet decomposition produces dyadic native spatial resolutions, related to the original size of the image by a factor $(1 / 2)^{k}$, where $k$ is an integer.

Few works in the literature have investigated the inclusion of spatial scalability by non-dyadic ratios with wavelet-based approaches. In [1], a post-processing stage is used in addition to a full decomposition tree obtained by extending the two-dimensional wavelet decomposition filter bank. While the scheme allows for dyadic and non-dyadic spatial scalability, the post-processing stage at the

A. Elmoataz et al. (Eds.): ICISP 2010, LNCS 6134, pp. 28 35, 2010.

(C) Springer-Verlag Berlin Heidelberg 2010 

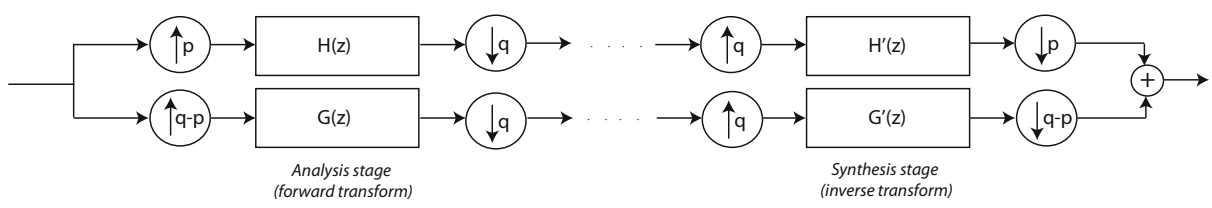

Fig. 1. A $(p, q)$ wavelet transform filter bank

decoder is an extra complexity and introduces additional distortion. In [2], $M$ band wavelet filter banks are exploited to produce spatial resolutions obtained by non-dyadic factors. The proposed method, however, requires a different set of synthesis filters to be built for every size ratio. In [3], a wavelet transform with sampling coefficients forming a non-integer ratio is proposed for generating nondyadic spatial resolutions; combining dyadic and non-dyadic spatial scalability within that framework was, however, not attempted.

In this paper, we propose using a combination of wavelet transforms of noninteger dilation factors capable of producing native spatial resolutions related to the original resolution by dyadic and non-dyadic ratios. Section 2 briefly reviews wavelet transform filter banks with rational dilation factors. In Section 3, the proposed subband decomposition is introduced and a constraint on the dilation factors is formulated to allow both dyadic and non-dyadic resolutions. An extension is presented in Section 4, and in Section [5] we describe a parent-child tree construction scheme for compatibility with zerotree-based subband coders. Finally, experimental results are presented in Section [6, and conclusions drawn in Section 7 .

\section{Rational Wavelet Transforms}

Fig. 1 illustrates a generalization of the two-band filter bank representation of a wavelet transform to a pair of integer sampling coefficients $p$ and $q$ with $p<q$. The ratio $q / p$ is defined as the dilation (or sampling) factor of the filter bank. The traditional definition of a wavelet transform, often qualified as dyadic, corresponds to a filter bank with sampling coefficients $(p, q)=(1,2)$ and a dilation factor $q / p=2$. When the sampling coefficients are not divisible, the filter bank is said to have a rational dilation factor and the wavelet transform is termed a rational wavelet transform. The interested reader is referred to [4, $[5]$ for a discussion on wavelet filter banks with rational dilation factors.

\section{Combined Rational Wavelet Decomposition for Spatial Scalability with Dyadic and Non-dyadic Ratios}

A two-dimensional wavelet decomposition is performed by applying the filter bank illustrated in Fig. 1] along the rows and the columns of the image, producing an approximation subband and three details subbands. Multiple levels of 


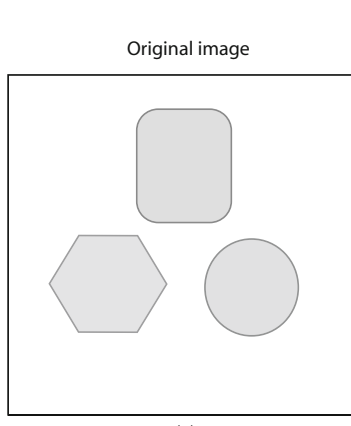

(a)

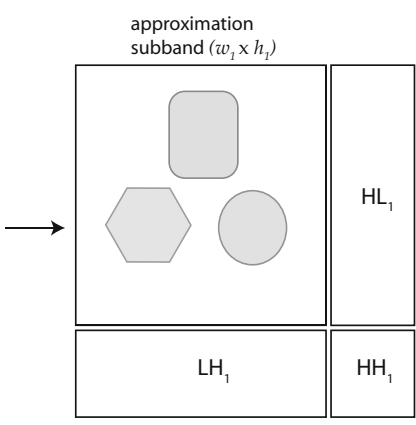

(b)

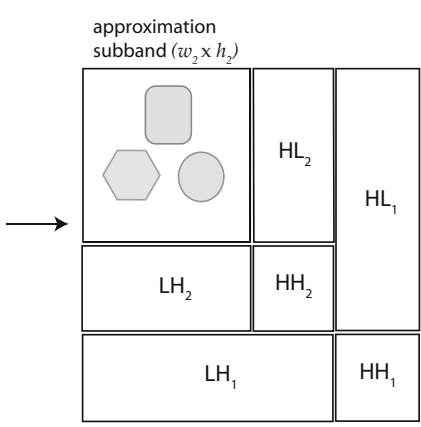

(c)

Fig. 2. Illustration of a two-dimensional combined rational wavelet decomposition: a $2 \mathrm{D}\left(p_{1}, q_{1}\right)$ wavelet transform is applied on the original image, then a $2 \mathrm{D}\left(p_{2}, q_{2}\right)$ wavelet transform is applied on the resulting approximation subband

decomposition are performed by recursively applying the 2D wavelet transform to the resulting approximation subband. The set of native spatial resolutions produced by a multi-level two-dimensional wavelet decomposition is completely determined by the size ratios between the successive sizes of the approximation subband and the size of the original image. If $\left(w_{0}, h_{0}\right)$ is the size of the original image, $n$ levels of $2 \mathrm{D}$ decomposition with a wavelet transform of dilation factor $q / p$ results in an approximation subband of size $\left(w_{n}, h_{n}\right)=\left(\left(\frac{p}{q}\right)^{n} \times w_{0},\left(\frac{p}{q}\right)^{n} \times h_{0}\right)$. Hence, with a dilation factor of $q / p$, the set of native spatial resolutions is determined by the set of size ratios $\left\{(p / q)^{m}\right\}$, with $m=0, \ldots, n$.

It is clear from this formulation that the two-dimensional wavelet decomposition, as defined, is not capable of producing a set of native spatial resolutions containing both dyadic and non-dyadic ratios, regardless of the value of the dilation factor $q / p$. However, a different set of native resolutions can be obtained if the multi-level wavelet decomposition alternates between wavelet transforms of different dilation factors, as illustrated in Fig. 2. Such an alternated use of rational wavelet transforms in a multi-level two-dimensional wavelet decomposition is what we denote a combined rational wavelet decomposition. In the rest of the document, this terminology will often be simplified to combined decomposition.

The above definition can be generalized to any number of different dilation factors $q_{k} / p_{k}$. However, without loss of generality, the notion of combined rational wavelet decomposition is described in the current paper for a combination of only two different rational wavelet transforms. Given an original image of size $\left(w_{0}, h_{0}\right)$, the combined decomposition illustrated in Fig. 2 produces native spatial resolutions of sizes $\left(w_{1}, h_{1}\right)=\left(\frac{p_{1}}{q_{1}} \times w_{0}, \frac{p_{1}}{q_{1}} \times h_{0}\right)$ and $\left(w_{2}, h_{2}\right)=\left(\frac{p_{1}}{q_{1}} \times \frac{p_{2}}{q_{2}} \times w_{0}, \frac{p_{1}}{q_{1}} \times \frac{p_{2}}{q_{2}} \times h_{0}\right)$, successively. This constitutes one level of combined decomposition. Multiple levels of combined decomposition are performed by reiterating the scheme on the approximation subband. It can be demonstrated that the set of native spatial resolutions produced by $n$ levels of combined decomposition is determined by the two sets of size ratios $\left\{\left(\frac{p_{1}}{q_{1}}\right)^{m}\left(\frac{p_{2}}{q_{2}}\right)^{m-1}, m=1, \ldots, n\right\}$ and $\left\{\left(\frac{p_{1}}{q_{1}}\right)^{m}\left(\frac{p_{2}}{q_{2}}\right)^{m}, m=0, \ldots, n\right\}$. From the latter 
list, we can establish that the set of native spatial resolutions will include resolutions obtained by dyadic ratios if the dilation factors are selected such that

$$
\prod_{i}\left(q_{i} / p_{i}\right)=2
$$

An example of dilation factors satisfying this condition is the pair $q_{1} / p_{1}=4 / 3$ and $q_{2} / p_{2}=3 / 2$. A two-level combined decomposition with wavelet transforms of dilation factors $q_{1} / p_{1}=4 / 3$ and $q_{2} / p_{2}=3 / 2$ produces the set of size ratios $\{1,3 / 4,1 / 2,3 / 8,1 / 4\}$. The corresponding native spatial resolutions are indicated in Table 1 for an original image at $4 \mathrm{CIF}$ resolution; the result is an extended set of native resolutions when compared to a regular dyadic wavelet decomposition.

Table 1. Native spatial resolutions from an original image of size $704 \times 576$

\begin{tabular}{|l|c|c|c|}
\hline & $n=0$ & $n=1$ & $n=2$ \\
\hline Dyadic wavelet decomposition & $704 \times 576$ & $352 \times 288$ & $176 \times 144$ \\
\hline $\begin{array}{l}\text { Combined decomposition with } \\
q_{1} / p_{1}=4 / 3 \text { and } q_{2} / p_{2}=3 / 2\end{array}$ & & & \\
\hline
\end{tabular}

\section{A Wavelet Packet Extension to the Combined Rational Wavelet Decomposition}

In this section, we propose an extension to the combined rational wavelet decomposition introduced in Section 3. The extension, denoted wavelet packet extension, consists of performing an additional one-dimensional wavelet transform on the horizontal and vertical subbands from the $\left(p_{1}, q_{1}\right)$ wavelet decomposition, using the $\left(p_{2}, q_{2}\right)$ wavelet transform. This is illustrated in Fig. 3 for the pair of dilation factors $q_{1} / p_{1}=4 / 3$ and $q_{2} / p_{2}=3 / 2$.

It can be observed in Fig. 3 that with this specific pair of dilation factors, the wavelet packet extension results in a subband decomposition similar to a wavelet packet decomposition obtained with the use of a dyadic wavelet transform. This similarity has a significant advantage: it translates in natural compatibility with a wide base of existing wavelet-based image coding frameworks. Indeed, since the support for alternative decompositions structures was added to Part 2 of the JPEG2000 image coding reference standard, many existing frameworks and subband coders developed for wavelet-based image compression have naturally included support for dyadic wavelet packet decomposition structures. Furthermore, with the described wavelet packet extension mode, dyadic wavelet decomposition and combined decomposition can be used together in a multi-level decomposition, and the resulting subband structure can be made compatible with existing subband coders by taking advantage of the similarity with dyadic wavelet packet decompositions. An example is illustrated in Section 5 for subband coders of the zerotree coding family. 


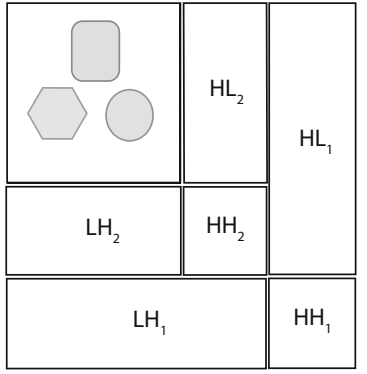

Subbands from one level of combined decomposition

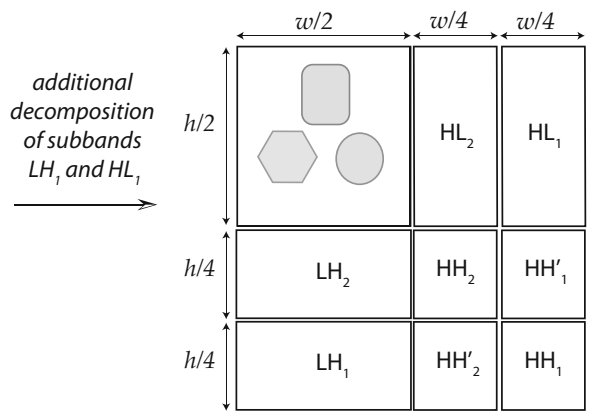

Fig. 3. Illustration of the wavelet packet extension for a combined decomposition with $q_{1} / p_{1}=4 / 3$ and $q_{2} / p_{2}=3 / 2$

\section{Adapting the Parent-Child Tree Construction Scheme for Zerotree-Based Subband Coders}

A natural challenge that arises from a new two-dimensional subband decomposition structure is the compatibility with existing subband coders. In this section, we address this issue for subband coders based on a zerotree parent-child structure, of which the widely-used SPIHT 6] is a good example. Zerotree-based subband coders use a parent-child tree structure built among the wavelet subbands. With a dyadic wavelet decomposition $(q / p=2)$, the construction of the parent-child tree takes advantage of the constant integer ratio of two between the sizes of subbands at consecutive levels of decomposition. In the case of a combined rational wavelet decomposition, an integer ratio in size is not always possible between subbands produced by wavelet transforms of different dilation factors. For example, considering the subband decomposition depicted in Fig. 2, and the pair $q_{1} / p_{1}=4 / 3$ and $q_{2} / p_{2}=3 / 2$, the ratio in size between the subbands $H L_{1}$ and $H L_{2}$ would be 1 along the width and $3 / 2$ along the height.

In order to overcome the issue of non-integer ratios, we propose to group subbands by levels of combined decomposition and associate any subband $\mathcal{S}^{k}$ obtained after $k$ levels of combined decomposition to the corresponding subband $\mathcal{S}^{k+1}$ obtained after $k+1$ levels. If the dilation factors are selected according to (1), the ratio in size between two subbands $\mathcal{S}^{k}$ and $\mathcal{S}^{k+1}$ at consecutive levels of combined decomposition is a constant integer value of two. From this derivation, we propose to adapt the parent-child tree construction scheme from the traditional method depicted in Fig. 4(a) to the manner illustrated in Fig. 4(b). The parallel between the two schemes is clearly visible. Fig. 4)(c) on the other hand illustrates how the parent-child tree can be built when multi-level combined decomposition and multi-level dyadic wavelet decomposition are used in conjunction. The illustrated method considers the special case where $q_{1} / p_{1}=4 / 3$, $q_{2} / p_{2}=3 / 2$, and the wavelet packet extension is used for the combined decomposition. The similarity with dyadic wavelet packet decomposition structures is 


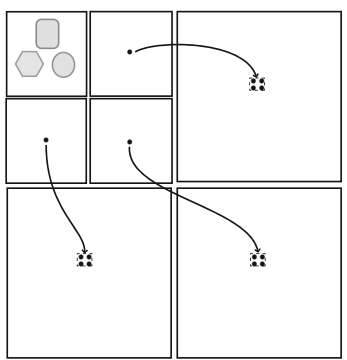

(a) two-level dyadic decomposition

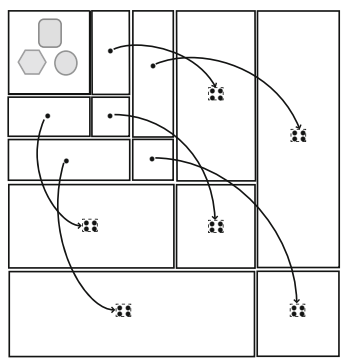

(b) two-level combined decomposition

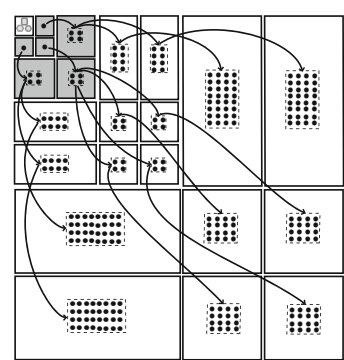

(c) two-level combined decomposition + two-level dyadic decomposition

Fig. 4. Adaptation of the parent-child tree construction scheme

taken advantage of, notably in the transition between the lowest level of dyadic wavelet decomposition and the highest level of combined decomposition.

\section{Experimental Results}

We implemented the combined decomposition with wavelet transforms of dilation factors $q_{1} / p_{1}=4 / 3$ and $q_{2} / p_{2}=3 / 2$ in a wavelet-based image compression system with support for spatial scalability. The subband coding stage of the compression is done with an adaptation of SPIHT [6] capable of producing resolution-scalable bitstreams [7], and added the ability to specify a bitstream length for every spatial resolution of interest in the bitstream. The encoded image is decoded at full size resolution and at $3 / 4$ the original size using the native spatial resolution available at the decoder.

A similar image coder is built using only dyadic wavelet decomposition. Because a native spatial resolution corresponding to $3 / 4$ the original size of the image is not available at the decoder, two configurations are defined:

1. the image is to be decoded at half-size resolution using the native resolution available, and then interpolated to $3 / 4$ the original size of the image.

2. the image is to be decoded at full size resolution, and then decimated to an image of $3 / 4$ the original size.

We denote these two configurations of the dyadic wavelet-based image coder respectively as B1 and B2, while A designates our image coder built with combined decomposition. Four levels of dyadic wavelet decomposition are specified for the image coders B1 and B2. The image coder A uses two levels of combined decomposition followed by two levels of dyadic wavelet decomposition; our experimentations have shown that this produces slightly better results than the use of four levels of combined decomposition. The CDF9/ 7 filters are used for the dyadic wavelet transforms, and the rational wavelet transforms are performed using wavelet filters of four vanishing moments obtained from [5]. Finally, for all three image coders, the subband coder imposes $3 / 4$ of the full bit budget to be assigned to the data needed to obtain the image at $3 / 4$ the original size. 

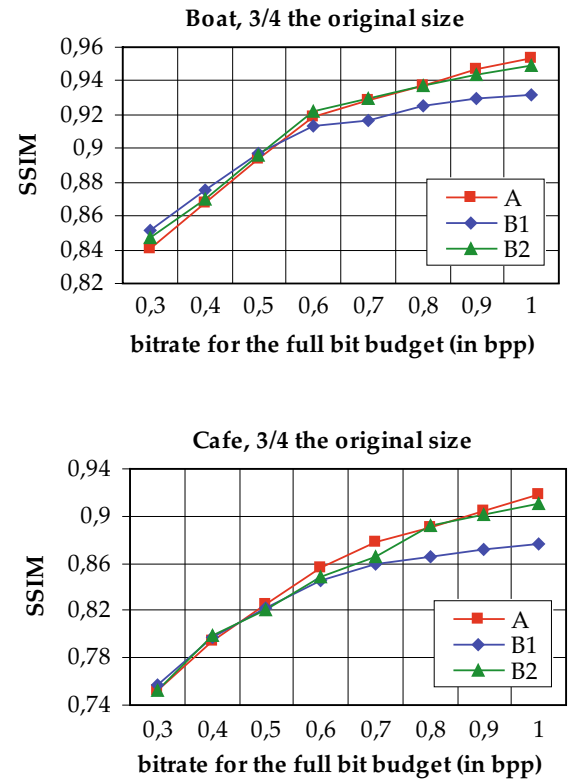
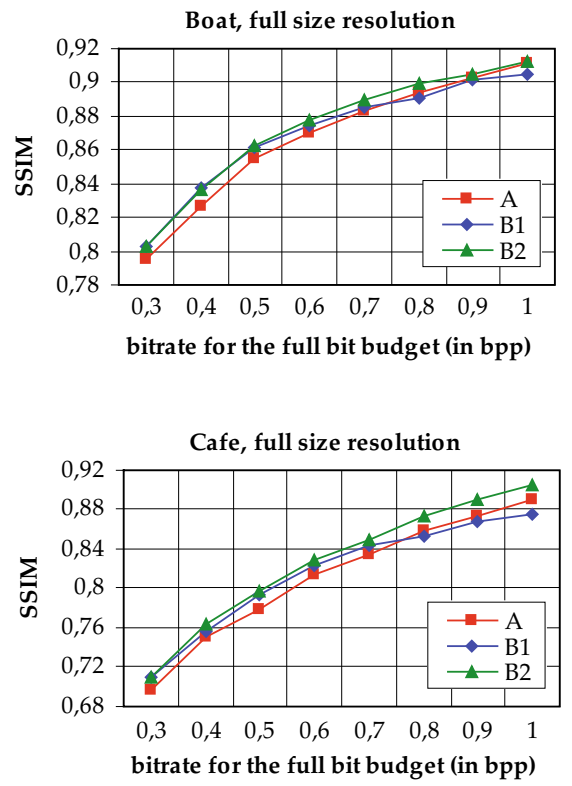

Fig. 5. Experimental results

Experiments are run with the images Boat of size $512 \times 512$ and Cafe of size $1024 \times 1280$ (a half-size version of the popular JPEG2000 test image). Bitrates for the full bit budget range from 0.3 to 1 bits per pixel, and the quality of the decoded images is measured using the Structural Similarity Index Metric (SSIM). A resized version of the original image is used as reference for images of lower spatial resolution. Bicubic interpolation/decimation is used to perform resizing operations for B1/B2 outputs.

Fig. 5 illustrates the objective quality scores obtained with the different sets of images. The results display a gain in quality when using the image coder with combined decomposition compared to the alternative of interpolating the lower dyadic resolution of a dyadic wavelet-based image coder. Furthermore, across the range of bitrates, visual quality remains competitive against the second alternative of decimating the higher dyadic resolution. Quality scores at full size resolution illustrate a tradeoff of having extended spatial scalability through combined rational wavelet decomposition, as the images decoded at full size resolution are shown to be of lesser quality than the outputs from the dyadic wavelet-based image coder. The apparent drop in quality at higher bitrates for full size images obtained from the image coder B1 can be explained by the fact that the subband coder assigns $3 / 4$ of the bit budget to the data needed for the half-size image used to obtain an image at $3 / 4$ the original size. 


\section{Conclusions}

In this paper, we have introduced a multi-level wavelet decomposition structure made of combining rational wavelet transforms of different dilation factors. We've shown that with a proper selection of dilation factors, the proposed decomposition allows for spatial scalability with dyadic and non-dyadic ratios, with minimal impact on coding efficiency. Future work on the subject will include a more extensive performance analysis with various sets of wavelet filters for the rational wavelet transforms, and a study on the impact of combining different wavelet transforms in a multi-level subband decomposition.

Acknowledgements. The authors would like to thank Nortel and the Natural Sciences and Engineering Research Council of Canada for funding this research.

\section{References}

1. Nakachi, T., Sawabe, T., Suzuki, J., Fujii, T.: A study on non-octave scalable image coding and its performance evaluation using digital cinema test material. IEICE Trans. Fundamentals E89-A(9), 2405-2414 (2006)

2. Pau, G., Pesquet-Popescu, A., Piella, G.: Modified $M$-band synthesis filter bank for fractional scalability of images. IEEE Signal Processing Letters 13(6), 345-348 (2006)

3. Xiong, R., Xu, J., Wu, F.: A lifting-based wavelet transform supporting non-dyadic spatial scalability. In: IEEE International Conference on Image Processing, October 2006, pp. 1861-1864 (2006)

4. Blu, T.: A new design algorithm for two-band orthonormal rational filter banks and orthonormal rational wavelets. IEEE Trans. on Signal Processing 46(6), 1494-1504 (1998)

5. Bayram, I., Selesnick, I.W.: Orthonormal FBs with rational sampling factors and oversampled DFT-modulated FBs: A connection with filter design. IEEE Trans. on Signal Processing 57(7), 2515-2526 (2009)

6. Said, A., Pearlman, W.: A new, fast, and efficient image codec based on set partitioning in hierarchical trees. IEEE Trans. on Circuits and Systems for Video Technology 6(3), 243-250 (1996), doi:10.1109/76.499834

7. Christophe, E., Pearlman, W.A.: Three-dimensional SPIHT coding of volume images with random access and resolution scalability. EURASIP Journal on Image and Video Processing 2008 (2008) 